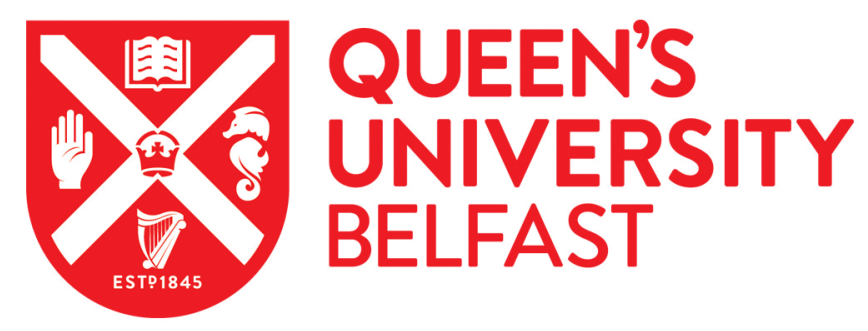

\title{
Constant-er Lens Beamformer for Low-Complexity Millimeter-Wave Hybrid MIMO
}

\author{
Abbasi, M. A. B., Fusco, V. F., Tataria, H., \& Matthaiou, M. (2019). Constant- $\varepsilon$ Lens Beamformer for \\ Low-Complexity Millimeter-Wave Hybrid MIMO. IEEE Transactions on Microwave Theory and Techniques, \\ 67(7), 2894. https://doi.org/10.1109/TMTT.2019.2903790
}

Published in:

IEEE Transactions on Microwave Theory and Techniques

Document Version:

Peer reviewed version

Queen's University Belfast - Research Portal:

Link to publication record in Queen's University Belfast Research Portal

Publisher rights

(C) 2019 IEEE. This work is made available online in accordance with the publisher's policies. Please refer to any applicable terms of use of the publisher.

\section{General rights}

Copyright for the publications made accessible via the Queen's University Belfast Research Portal is retained by the author(s) and / or other copyright owners and it is a condition of accessing these publications that users recognise and abide by the legal requirements associated with these rights.

Take down policy

The Research Portal is Queen's institutional repository that provides access to Queen's research output. Every effort has been made to ensure that content in the Research Portal does not infringe any person's rights, or applicable UK laws. If you discover content in the Research Portal that you believe breaches copyright or violates any law, please contact openaccess@qub.ac.uk. 


\title{
Constant $-\epsilon_{r}$ Lens Beamformer for Low-Complexity Millimeter-Wave Hybrid MIMO
}

\author{
M. Ali Babar Abbasi, Member, IEEE, Vincent F. Fusco, Fellow, IEEE, Harsh Tataria, Member, IEEE, and \\ Michail Matthaiou, Senior Member, IEEE
}

\begin{abstract}
It is well established that the utilization of unused millimeter-wave (mmWave) spectrum is inevitable due to unavailability of required bandwidth in the conventional RF band to support the high data demands of 5G. Large antenna arrays with beamforming capabilities are required to compensate for the high path-loss at mmWave frequencies. We are at the verge of a massive mmWave radio front-end deployment and low-complexity low-cost hardware beamforming solutions are required now at this stage than ever before. In this work, one such solution is demonstrated and analyzed. A high performance and low-complexity lens based beamformer consisting of constant dielectric material $\left(\epsilon_{r}\right)$ with antenna-feeds is presented for multibeams operation. A prototype is developed based on the classical synthesis approach, and in line with the requirements of mmWave hybrid multi-user multiple-input multiple-output (MU-MIMO) systems. A characterization at $28 \mathrm{GHz}$ is performed wherein uplink signal-to-noise-ratio of user terminals is evaluated with the zero-forcing (ZF) baseband signal processing. Radiation performance of a single source beamformer is measured in an anechoic environment and end-to-end ergodic sum spectral efficiency performance is estimated based on the measured data. It is shown that the constant- $\epsilon_{r}$ based beamformer solution is simple, yet significantly outperforms conventional antenna array beamformers with analog phase shifter network, making it a promising candidate for future hybrid massive MIMO systems.
\end{abstract}

Index Terms-Beam forming, lens, millimeter-waves, MIMO, MU-MIMO, spectral efficiency, 5G

\section{INTRODUCTION}

$\mathrm{D}$ URING the last decade, we have witnessed an enormous evolution in commercial wireless communication systems, from simple voice and messaging services to data hungry mobile broadband. End user data rate is expected to grow even further and current cellular infrastructure is in no position to meet these formidable demands. Researchers are putting together efforts in finding novel approaches to further improve the overall throughput of the wireless systems. By the end of 2020 we are expecting a data rate 10 times compared to 2010. Multiple-input multiple-output (MIMO) are posed to meet the expected spectral efficiency demands by providing a higher capacity gains and a better link reliability. Lack of wide spectrum at conventional cellular a.k.a. sub-6 GHz, frequencies has compelled a migration towards millimeter-waves

This work was supported by the UK Engineering and Physical Science Research Council (EPSRC) under Grant EP/P000673/1 and EP/N020391/1.

M. A. B. Abbasi, V. F. Fusco, and M. Matthaiou are with the Institute of Electronics, Communications and Information Technology (ECIT), School of Electronics, Queen's University Belfast, Belfast BT3 9DT, United Kingdom.

$\mathrm{H}$. Tataria is with the Department of Electrical Engineering, Lund University, Lund, Sweden. (emails: m.abbasi@qub.ac.uk, v.fusco@ecit.qub.ac.uk, harsh.tataria@eit.lth.se,m.matthaiou@qub.ac.uk)
(mmWave); however, in order to reap the real potential of mmWave MIMO systems, a number of fundamental challenges are yet to be addressed. First and foremost, the propagation characteristics of a mmWave radio channels relatively different to the sub-6 GHz channels. The free space path-loss of a radio wave at mmWave frequencies requires compensation in terms of gain, and in order to do this, multiple-antenna beamforming is a necessity. Many of the specialized telecommunication research groups and wireless equipment vendors are putting together their efforts in an attempt to propose the most suitable specialized hybrid transceiver architectures [1]-[4]. The objective is the inclusion of mmWave to the future cellular infrastructure enabling 5G, and beyond. Theoretically, mmWave massive MIMO is found to surpasses other technologies in this respect [5], however, practical mmWave radio-frequency (RF) front-end is complex and expensive. In addition to reducing the this complexity, the throughput per square meter needs to be increased to meet the unprecedented projected increase in data rate demand. Shrinking the cell size with the associated interference mitigation is considered to be one possible approach. With this, the requirement of hardware increases, so do the deployment cost. A lowcomplexity and low-cost mmWave radio front-end hardware with beamforming capabilities, supported by a hybrid MIMO for small cell size can be the answer. In this paper we propose one such solution. In particular, our focus is on synthesis and characterization of a low-complexity constant $-\epsilon_{r}$ lens based beamformer. We evaluate the performance of the lens with the help of theoretical principles, real-time measurements and electromagnetic/numerical simulations. Unlike gradient refractive index material based, and metamaterials based lenses, the implementation of the presented solution is simpler and effective. Taking into account the overall framework for the multiuser communication system, we then integrate the lens with the hybrid architecture. We consider the RF switching, finite numbers of RF chains, and zero-forcing (ZF) baseband signal processing (SP) to predict the end-to-end uplink performance. A detailed insight into the engineering trade-offs of the proposed architecture is discussed.

\section{LENS BASED BEAMFORMERS}

Hybrid antenna array beamformers comprising of a phase shifter network demonstrate high directivity and beam scanning, however, their practical implementation at mmWave frequencies is complex and expensive. Lens based beamforming systems are found to be a lucrative alternative [6]. In spherical 
or semi-spherical lenses, a beam traveling along the principle axis of the lens is focused to a spot, known as the focal point. Conversely, an electromagnetic radiation source placed at the focal point can convert into a directional beam by the lens, so an object at the focal point has an image at infinity, and vice versa. There always exist anomalies in lenses such as coma, chromatic, spherical aberration, astigmatism and so on, caused because of lens imperfections. This limits the focusing abilities of lenses. One of the most common type of lens used at the radio frequencies is the Luneburg lens, originally proposed in [7]. Variation of similar lens type are generally designed based on transformation optics theory. By carefully grading the refractive index of the lens material, different focusing properties can be achieved. One of such gradient index principle is given

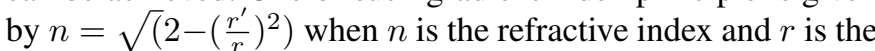
radius of the sphere. For such spherical lenses, the diameter is inversely proportional to the achievable half power beam width (HPBW). Theoretical conversion of 3-dimensional (3D) lens action on 2-D surface has also been demonstrated via focusing beam in a limited azimuthal or elevation planes [8]. Lundberg lens principle can be re-created by varying the parallel plate spacing along the radius of a circular lens in [9], where a slot antenna is used to feed the lens and air is used as dielectric. In a recent studies, a 2-D slab based lens with field control capabilities to achieve similar graded index principle is shown [10]. Beamforming has also been achieved using 3-D monolithic low-loss periodic structures with uniform $\epsilon_{r}$ [11]. Another two-layered metamaterial planar lens is shown in [12], that uses a linear substrate integrated waveguide (SIW) antenna array for spatial beamforming. It is shown that the wave impedance can be controlled in the structure by geometrical adjustments on a periodic lattice. A special class of metasurfaces composed of densely packed thin planar arrays, utilizing the physics of subwavelength resonant material elements, are found to focus the EM energy in a similar fashion as a lens does [13]. Similar 3-D mertasurfaces based lens is realized in [14] machined by drilling inhomogeneous holes in multilayered dielectric plates. The design strategy shows a successful reduction in the reflection and insertion losses of the lens at mmWaves. The capabilities of lenses can further be advanced by adding polarization manipulation, multi-band operation, frequency agility, sometimes amplification [15] using advanced and more sophisticated design approaches. Devising of all the aforementioned lenses is a complex task and in addition to fundamental design variations, they sometimes require reconfigurability within the lens main structure to realize the propagating wave manipulations and phase shifting. A comparatively easier approach of realizing the lens operation using transmission line theory, known as Rotman lens, is also well known. The simplification of such lens type stems from using standard photolithography method, widely used for rapid circuit boards fabrication. A Rotman lens with a compact antenna array and integrated amplifiers at $60 \mathrm{GHz}$ is shown to be capable of beam-steering and active beam-switching at the same time [16]. Both these functions are essential for hybrid MIMO operation [6]. Another similar SIW based Rotman lens is presented in [17] capable of 7 beam selection using SIW antenna array. A thorough investigation of Rotman lens based MIMO system with beam selection and digital beamforming is reported in [18]. Regardless of Rotman's, Fourier's or Luneburg's theoretical synthesis approaches, lens arrays are generally found to be much effective in simplifying the mmWave MIMO radio freuency (RF) front-end. Moreover, it has also been shown that lens antenna arrays are effective in simplify the signal processing required to achieve high data rates with multiple antennas [19]. This is done by exploiting the mmWave channel angular sparsity, even when channels are frequency selective.

At sub-6 GHz frequencies, especially at below $1 \mathrm{GHz}$, the size of a lens based beamformer becomes large and impractical compared to other similar antenna types with same desirable specifications. At mmWave frequencies, the only constraint i.e. size, is no more a problem [20]. One can argue that the size of a lens at mmWave frequencies is more practical compared to lower frequencies since it offers a high directivity as well as a fairly controllable integration with the mmWave electronics. Beamformers for cellular application need an outdoor deployment where weather conditions, material aging and thermal expansion play a vital role. This makes the gradient $\epsilon_{r}$ lenses, like the Luneburg, not the best choice since the maintenance of gradient $\epsilon_{r}$ requiring layering of varying refractive index material is problematical. A constant$\epsilon_{r}$ lens is capable of solving these issues. The synthesis approach and electromagnetic analysis of such lens type is available in classical literature [21]-[23]. Constant dielectric lens operation at the mmWave spectrum is shown in a number of studies [24]-[28]. Full spherical lens with a mmWave feed is shown to be a reliable source of multibeam operation. Hemispherical or grooved constant dielectric lenses are other similar ways to enhance the feed antenna gain. However, to analyze the rightfulness of such lens types for the high-data rate communication is yet to be shown. Also, in addition to the low-complexity prospective of such lens types, the scalability factor of the RF front-end hardware is missing from the literature to the best of author's knowledge. In the work, we fill this gap by showing the end-to-end sum spectral efficiency performance of constant $-\epsilon_{r}$ lens for the mmWave hybrid multi-user MIMO (MU-MIMO) uplink. The constant$\epsilon_{r}$ lens is constructed from a single plastic billet and has the property that the focal point can be made to lie just off the surface of the lens. This latter aspect makes sampling of the rays for further beamforming considerably simpler than by other methods. Devising a lens with a single dielectric material not only decreases the implementation complexity and manufacturing cost, but also gives a full control on the choice of the material. Modern plastics exhibit low mmWave losses, are homogeneous, and do not readily age or absorb moisture [29]. The radiation performance of the constant$\epsilon_{r}$ lens is comparable to planar array configurations like the ones presented in [6], [30]-[31], with comparatively marginal deployment complexity.

\section{HyBRID MIMO ARCHITECTURE}

\section{A. System Model}

The mmWave MIMO model considered in this study is a typical uplink multiuser system in a single-cell with a radius 
of $K_{\text {sector. }}$ A base station (BS) is located at the origin of the cell. To perform a comprehensive comparison, we separately considered the BS to be equipped with a $M$ element ULA beamformer, a URA beamformer and a constant $-\epsilon_{r}$ based beamformer. The beamforming mechanism in both ULA and URA is governed by the Rotman lens based passive phase shifters [18], [32]. Description on the URA and ULA are discussed is detail in [33]-[35]. Although MIMO operation is also expected at mmWave user equipment (UE), for the sake of simplicity, in this study we assume single-antenna terminals. UEs send $L$ independent data streams within the same timefrequency resource with a uniform uplink power. We assumed a uniform distribution of UEs within a discrete sector of $100^{\circ}$ in azimuth for the case of ULA beamformer. In case of URA and constant $-\epsilon_{r}$ lens beamformer, we added an elevation sector of $30^{\circ}$ in the simulations. ${ }^{1}$ To keep our analysis simple, we assume that the BS has perfect knowledge of the propagation channels.

Remark 1. The assumption of perfect channel knowledge may at first sight may seem rather naive. However, there are several fundamental reasons for this: Firstly, unlike previous studies, the central focus of our work is on aggressive RF circuit reduction techniques to lower the implementation complexity of mmWave hybrid beamforming, where we propose a modification on the conventional architecture with a Rotman lens-based beamformer. Here, the assumption of perfect channel knowledge is necessary in order not to obfuscate the findings from the aforementioned study. Secondly, in line with [36], this assumption is reasonable in scenarios with low terminal mobility, where a large fraction of the channel coherence time can be spent for accurate channel acquisition at the BS. Thirdly, the results obtained from subsequent evaluations can be treated as a useful upper bound on what may be achieved in practice, with imperfect channel estimates at the BS.

The method of receiving and processing the direction-ofarrival (DOA) in all three considered beamformers is different. For an ideal lens, the core objective is to simultaneously provide power combining and phase shifting in order to focus the incoming energy to a specific RF port. In the case of ULA, the Rotman lens is responsible of this function as it delivers $L$ DOAs to the beam-port outputs. The only difference in URA beamformer, compared to ULA, is that the output beam-ports of the Rotman lens connected to URA includes both azimuth and elevation coverage zone DOAs. On the other hand, the beam forming mechanism is happening at the electromagnetic domain in the case of constant $-\epsilon_{r}$ lens, the DOAs are directly delivered to the horn-feed output. The constant- $\epsilon_{r}$ lens with the horn-feed conforms to the simplest approach i.e. double convex lens in [31]. Following the conventional hybrid mmWave MIMO topologies, the output of all beamformers is then connected to a $L \times M$ network of RF switches. The main purpose of the RF switch network is to ensure selection of $L$ non-overlapping and independent streams, out of $M$ possible outputs from the beamformer. After the RF switching, the

\footnotetext{
${ }^{1}$ The $100^{\circ}$ azimuth and $30^{\circ}$ elevation sector is defined from $\left[-50^{\circ},+50^{\circ}\right]$ and $\left[-15^{\circ},+15^{\circ}\right]$ from the reference axis at the array broadside direction $0^{\circ}$, respectively.
}

remainder of the down conversion chains in this study is assumed to be perfect, since after beam selection, $L$ independent data streams have to have a dedicated RF chain [33]. After the down conversion, zero-forcing is employed to separate the gain and phase of $L$ data streams. Assume the beamformer to be capable of creating $M$ fixed analog beams along azimuth and elevation sectors as $\left(\phi_{1}, \theta_{1}\right),\left(\phi_{2}, \theta_{2}\right), \ldots,\left(\phi_{M}, \theta_{M}\right)$. The dimension-reduced $L \times 1$ signals after the switching matrix is given by

$$
\mathbf{y}=\rho_{\mathrm{t}}^{\frac{1}{2}} \mathbf{S}_{\mathrm{RF}} \mathbf{F}_{\mathrm{RF}} \mathbf{H x}+\mathbf{n} .
$$

Boldface upper case symbols represents matrix while the lower case symbols are vectors. The average transmit power of each terminal is denoted by $\rho_{\mathrm{t}}$, when $\mathbb{E}\left[\left|x_{\ell}\right|^{2}\right]=1$ and $\forall \ell=1,2, \ldots, L$, where statistical expectation and scalar norm operations are denoted by $\mathbb{E}$ and $|\cdot|$, respectively. Also, $\rho_{\mathrm{t}}^{1 / 2} \mathrm{x}$ is the payload data in uplink, formulated as $L \times 1$ vector. Here the additive Gaussian noise is modeled such that each entry of $\mathbf{n} \sim \mathcal{C N}(0,1) . \mathrm{S}_{\mathrm{RF}}$ is the switching network represented by a binary matrix, when it only have one non-zero entry in a row that corresponds to the selected beam index. Also, $\mathbf{G}=\mathbf{S}_{\mathrm{RF}} \mathbf{F}_{\mathrm{RF}} \mathbf{H}=\left[\begin{array}{llll}\mathbf{g}_{1} & \mathbf{g}_{2} & \ldots & \mathbf{g}_{L}\end{array}\right]$ is an $L \times L$ matrix comprising of $L$ vectors. Here $\mathbf{g}_{\ell}=\mathbf{S}_{\mathrm{RF}} \mathbf{F}_{\mathrm{RF}} \mathbf{h}_{\ell}$, $\forall \ell=1,2, \ldots, L$ [35]. We use a double-directional uplink channel model $\ell$-th terminal described in [37], [38] with finite multipath components (MPCs) at $28 \mathrm{GHz}$. The model assuming $N_{\mathrm{P}}$ MPCs is described by:

$$
\mathbf{h}_{\ell}=\frac{1}{\sqrt{N_{\mathrm{P}}}} \sum_{p=1}^{N_{\mathrm{P}}} \alpha_{\ell, p} \Lambda\left(\phi_{\ell, p}, \theta_{\ell, p}\right) \mathbf{a}^{H}\left(\phi_{\ell, p}, \theta_{\ell, p}\right),
$$

where $\alpha_{\ell, p}$ defines the gain of the $p$-th MPC for the $l$ - uplink channel. For the case of URA and ULA, the per-antenna element gain is represented by $\Lambda\left(\phi_{\ell, p}, \theta_{\ell, p}\right)$, while the farfield steering vector is denoted by $\mathbf{a}\left(\phi_{\ell, p}, \theta_{\ell, p}\right)$. Equation (2) is used for the ULA case, where isotropic antenna elements were assumed. On the other hand for the case of URA and constant$\epsilon_{r}$ based beamformer, instead of a classical description of an array gain defined by the product of per-antenna element gain and array factor, measured data is included discussed a the later stage.

\section{B. Constant $-\epsilon_{r}$ Lens Beamformer}

Due to large size compared to $\lambda$, collimated beam with ray tracing approach can be implemented to define the controlling parameters of a constant $-\epsilon_{r}$ lens. In Fig. 1, we consider a mmWave point source at location $A$. To create a constant phase plane wavefront parallel to contour $D E$ in a mmWave channel, the path length along the diameter $A E$ needs to be the same as $A C+C D$. This can be possible when the overall path length of the propagating wave along the $A C+C D$ is greater than the path length along $A E$ such that

$$
\sigma=A E \sqrt{\epsilon_{r}}-\left(A C \sqrt{\epsilon_{r}}+C D\right),
$$

where $\sigma$ defines the deviation. Normalizing (3) w.r.t to diameter of the lens gives: 


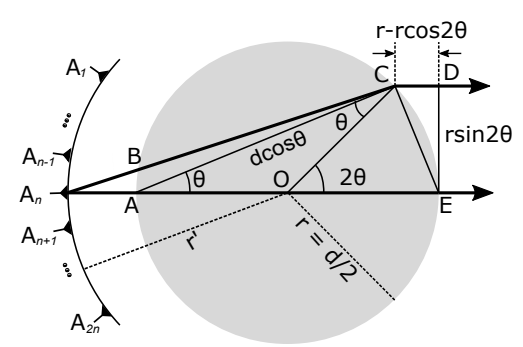

Fig. 1. Geometry of the constant $-\epsilon_{r}$ Lens.

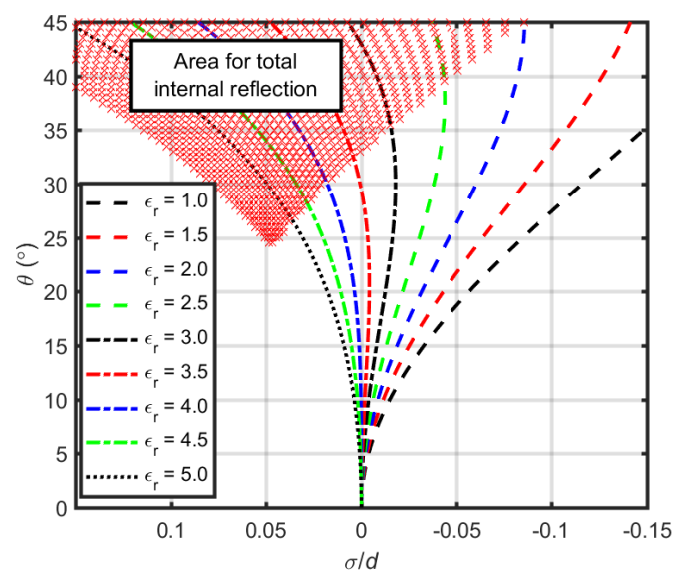

Fig. 2. Normalized deviation factor for a constant $-\epsilon_{r}$ Lens.

$$
\frac{\sigma}{d}=2 \sqrt{\epsilon_{r}} \sin ^{2} \frac{\theta}{2}-\sin ^{2} \theta
$$

All the sets of rays following a path $A C$ and leaving the lens surface thus defines the effective aperture area of the lens, given by:

$$
2 \times D E=d \times \sin 2 \theta .
$$

It can be noticed from Fig. 2 that the normalized deviation from a plane wavefront parallel to $D E$ is higher with high $\theta$. As $\epsilon_{r}$ increases from 1 to 5 , the deviation decreases with the lowest value when $\epsilon_{r} \approx 3.5$. Further increase in $\epsilon_{r}$ will cause the rays after a certain $\theta$ to exhibit total internal reflection defined by:

$$
\theta>=\sin ^{-1}\left(\frac{1}{\sqrt{\epsilon_{r}}}\right) .
$$

The effective aperture area of the lens is thus defined by the largest value of $\theta$ before which the ray from the point source A exhibits total internal reflection. The effective aperture area can also be postulated to decrease with an increase $\epsilon_{r}$. Therefore, the lens's material $\epsilon_{r}$ is bounded by the two choices. One is the maximum utilizable lens spherical area when $\epsilon_{r} \approx 2.0$ and $\theta \approx \pm 45^{\circ}$. The second choice is to use the $\epsilon_{r}$ required for minimum deviation factor, creating an efficient plane wavefront at the cost of limiting the usable lens surface aperture bounded by $\theta \approx \pm 32.5^{\circ}$. Based on this, it can be established that suitable lens operation is feasible for $2.0<\epsilon_{r}<3.5$. Approximately similar conclusions hold if the source is moved from point $A$ to a point $A_{n}$ outside the lens sphere provided that $A A_{n}<<A E$.

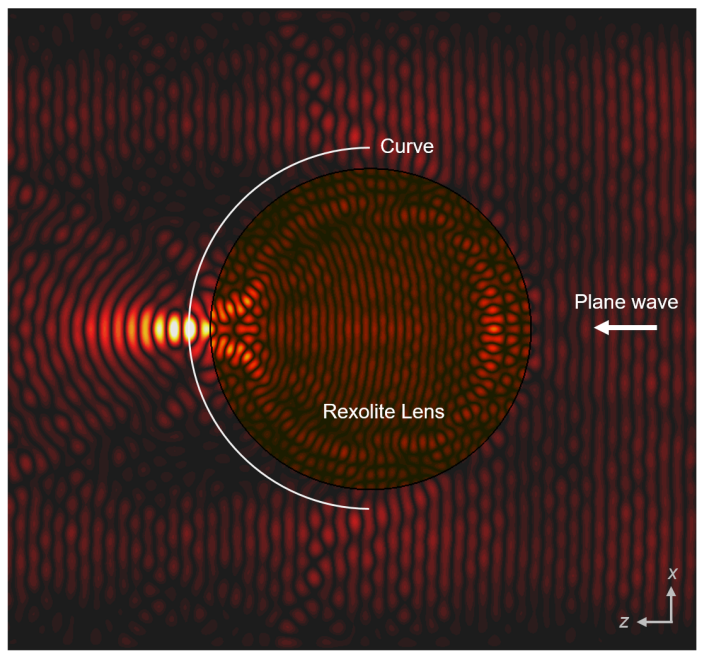

(a)

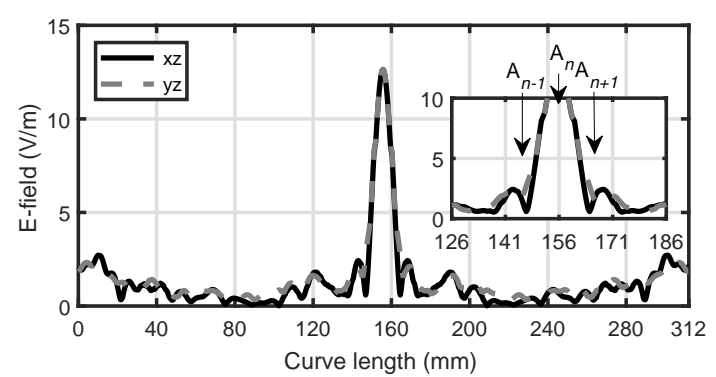

(b)

Fig. 3. (a) Simulated instantaneous real $E$-field strength with the plane wavefront excitation of constant $-\epsilon_{r}$ lens in full-wave EM solver (color map scale from 0 to $15 \mathrm{~V} / \mathrm{m}$ ). (b) Field strength along the curve.

Following this condition, $A_{n}$ outside the lens at the Petzval curvature [39], offers constant $-\epsilon_{r}$ lens another advantage over Luneburg's configuration with respect to the scalability of MIMO. However, it should be noted that for $d>\lambda$, propagation loss within the lens increases. From Fig. 2, it can be noticed that an incoming plane wavefront parallel to $D E$ cannot be focused at a single point. To further elaborate, take for example lens with $\epsilon_{r}=3$. Ideally, focusing on a single point means $\sigma / d=0$ throughout the usable range of $\theta$ (i.e. from $0^{\circ}$ to $35^{\circ}$ ). However, practically this is not the case since the $\epsilon_{r}=3$ contour is curved. Hence, the constant $-\epsilon_{r}$ lens will face the fundamental defect of aberration. It will be shown at a later stage that even when the focusing capability of a constant $-\epsilon_{r}$ lens is non-ideal, a large aperture area feed located at point $A$ can mitigate this defect. For example, a horn antenna-feed can handle a larger $d / \lambda$ ratio compared to a wire antenna-feed. Also, the homogeneity of $\epsilon_{r}$ is relative, so an inhomogeneous material can be considered homogeneous when the spatial variations occurring within the medium are, at a scale, smaller than $\lambda$.

A proof-of-concept constant $-\epsilon_{r}$ lens is realized using Rexolite [29] with $\epsilon_{r}=2.53$. Other material properties like density $=1.05 \mathrm{~g} / \mathrm{cm}^{3}$, dispersion factor $=0.00066$ and low coefficient of linear thermal expansion $=3.8 \times 10^{-5}$ in. $/$ in. $/{ }^{\circ} \mathrm{F}$ make Rexolite a very good choice for lens prototyping. Initially, 


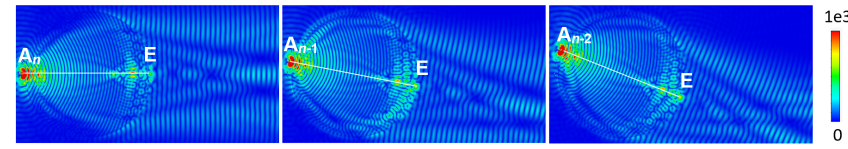

Fig. 4. Instantaneous electric field strength $x z$ plane at phase $=0^{\circ}$ when a patch antenna-feeds are placed along the curve.

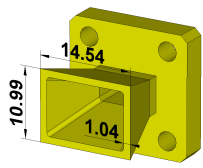

(a)

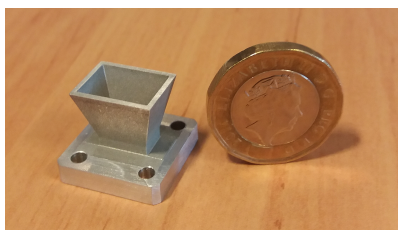

(b)
Fig. 5. (a) Geometrical configuration of horn antenna-feed for the constant$\epsilon_{r}$ lens (all dimensions are in $\mathrm{mm}$ ). (b) Horn antenna-feed machined from an aluminum block.

a standard patch antenna operating at $28 \mathrm{GHz}$ with free space gain of $6.45 \mathrm{dBi}$ was used as a radiation source at $A_{n}$. This antenna was re-optimized to operate efficiently in the presence of closely placed lens. To realize multi-beam MIMO operation, multiple antennas were placed along the first antenna in a geodesic fashion. The choice of neighboring antenna element location is important for two main reasons. First, to provide a spatial integrity, de-correlation between the beams is important. Secondly, a high resolution azimuth and elevation zone coverage requires the feed elements to be closely spaced, hence minimum mutual coupling is desirable. Note that the neighboring element in a standard planar antenna array formulation (i.e. $\lambda / 2$ ) is not required in this case since antenna array based beamforming is not desirable anymore. Also due to the geodesic placement of elements, uniform element spacing cannot be guaranteed along the spherical hemisphere of the lens. To evaluate the best possible neighboring element placement, first, the Rexolite lens is placed in 3-D Cartesian space. The antenna-feed location $A_{1} \ldots A_{2 n}$ is represented by Petzval curve in the $x z$-plane as shown in Fig. 3(a). A similar curve is placed along the $y z$-plane. The entire arrangement is then illuminated by a plane wavefront $E$-polarized along $y$-axis and propagating along $+z$-axis in CST Microwave Studio [40]. The instantaneous $E$-field along the observation plane $x z$ depict the field focusing capability of the lens. The absolute $E$-field along the curve is recorded (see Fig. 3 (b)) where E-field minimas are observed. Mutual coupling between elements can be reduced if the neighboring elements $A_{1} \ldots A_{2 n}$ are strategically placed in these $E$-field nulls. Such an arrangement allows a maximum of 81 patch antenna-feeds placed to host 81 beams using same Rexolite lens when $r^{\prime}=77.6 \mathrm{~mm}$ outside a lens with $d=133$ $\mathrm{mm}$. Increasing this number further will result in radiation performance degradation originated from the antenna-feed placed at $A_{n}$.

Remark 2. It can be mistakenly conjectured that the $E$ field minimas are the only best element placement options. In reality, a wide range of literature for mutual coupling reduction between closely spaced antenna elements can be integrated within the current model.

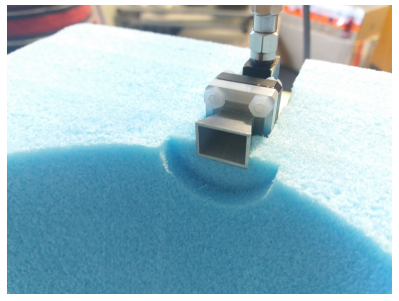

(a)

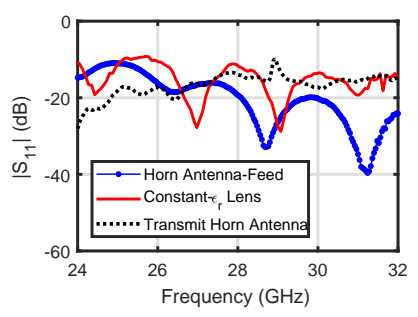

(b)

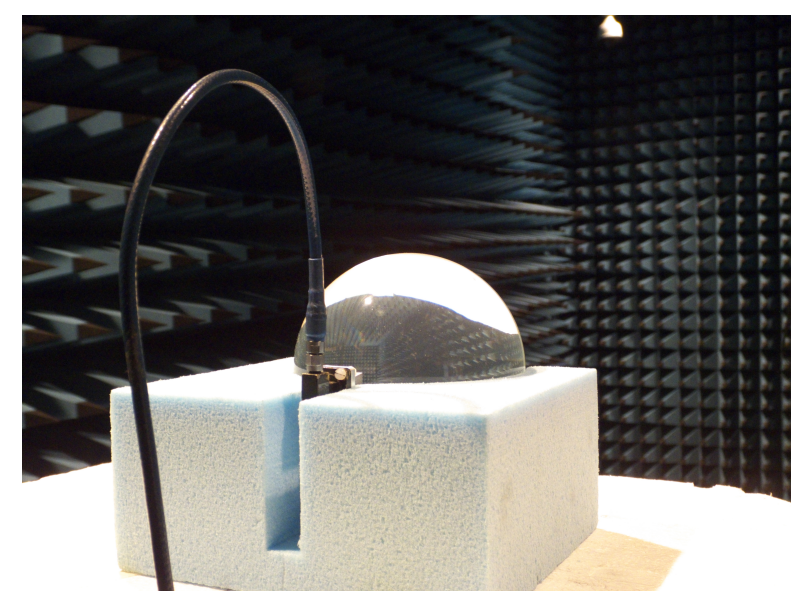

(c)

Fig. 6. (a) Assembly holder to ensure a precise spacing between horn antennafeed and the constant $-\epsilon_{r}$ lens. (b) Measured matching conditions before the measurements. (c) Measurement setup in NSI anechoic chamber.

The theoretical investigation based on ray-tracing cannot entirely reveal the physical limitations of the system when aperture antennas are used to feed the lens. There are two main reasons for that. First, at a small distance $A_{n} A$, the presence of Rexolite material in the near field of the antenna-feed makes its radiation properties inconspicuous. Secondly, contrary to the EM waves generated from a point source that theoretically have a pure spherical wavefront, the field oscillations within the practical antenna located at $r^{\prime}$ may not be able to generate proper continuous closed loops of electric field. Consequently, the EM energy entering the lens (from points $A$ or $B$ ) can either have a spherical or planar wave front depending upon the antenna-feed's radiation mechanism. Both of these reasons are evident from the $E$-field plots in Fig. 4. Here, a compact patch antenna-feeds (practical counterpart of a point source radiator) at $A_{n}, A_{n-1}$ and $A_{n-2}$ are simulated in the presence of other two feeds. As expected, the lens converts nearly spherical wavefronts to a plane wavefront, as the wave leaves the lens surface. As a result, a highly directive beam with a peak gain of $\sim 27 \mathrm{dBi}$ is achieved. The first side-lobe level relative to peak is $-12.5 \mathrm{~dB}$, while the $\mathrm{HPBW}$ stayed $<4.3^{\circ}$. It can be noticed from Fig. 4 that due to the spherical surface of the lens, the EM energy leaving the lens surface close to the aperture edge will face refraction, resulting in a high power density per unit area.

\section{BEAMFORMER HARdWARE AND MEASUREMENTS}

The analysis in previous sections narrows down the beamformer hardware trade-offs to four variables. First, the zone 
coverage resolution, governed by the system model. Second, the maximum focused radiation collection at a given DOA. Third, the neighboring antenna-feed placement at the $E-$ field minimas, can potentially reduce mutual coupling. Finally, realizable physical antenna-feed apertures ensuring geodesic element placement. In line with all four, we use the lens and horn antenna-feed. A spherical section with $d=133 \mathrm{~mm}$ was first machined out from a cylindrical block of Rexolite using a Triumph Dulex milling machine. Sphere was then carefully polished using Tech-Gen finisher to ensure surface roughness of the order of at most $<\lambda / 8$. The horn antenna-feed is followed by a WR28 waveguide capable of low insertion signal transmission within the band from 26.50 to $40 \mathrm{GHz}$. The antenna and waveguide was fabricated using an aluminum block of volume $20 \times 20 \times 20 \mathrm{~mm}^{3}$ first by VMC1000B CNC Vertical Machining Center. Precision detailing was then performed by Makino's Wire EDM facility. Dimensions and a photograph of the fabricated aluminum horn antenna-feed are presented in Fig. 5. The discussion in Section III-B, specifically around Fig. 3, concluded that wide aperture-feed is better to capture maximum EM evergy from point $A_{n}$. In line with this, a horn-feed is designed, simulated and optimized to capture maximum focused energy at a given DOA. Co-polarized $E-$ field component along the principle plane is evaluated in the $x y$-plane, tangential to the Petzval curvature in Fig. 3(a), and placed at the focal point $A_{n}$. The total area covered by the horn-feed opening overlaps $\sim 92 \%$ of the total electric field per unit area, relative to the total E-field outside the hornfeed area. The gain measurements of the lens were carried out in Anechoic chamber using standard techniques at $28 \mathrm{GHz}$. A standard gain horn antenna was used at the transmitter, while the lens with horn antenna-feed was placed in receiving mode. As mentioned before, spacing $A A_{n}$ is extremely important for constant $-\epsilon_{r}$ lens operation, a precise lens and horn antennafeed holder was machined and used for measurements (Fig. 6 (a)). The return loss of the transmitter horn and device under test (DUT) is shown in Fig. 6 (b). The entire assembly is connected to a rotation platform when axis limits are from $90^{\circ}$ to $90^{\circ}$. Laser alignment was used to align the transmitter and the receiver. A photograph of the measurement setup is shown in Fig. 6 (c). The point of peak directivity was assumed to be the reference point where azimuth and elevation are considered $0^{\circ}$. Originally, the maximum directivity point was captured at $+2^{\circ}$ elevation and was corrected at a post processing step. This error can be associated with the turn table level at the anechoic chamber. Measured high directivity beam is presented in Fig. 7(a), while the corrected measured patterns in 2-D plot are compared with the simulated predictions in Fig. 7(b). It is worth mentioning that a patch antenna was used initially as a practical approximation of a point source. Geodesic placement of patch antenna around the lens can also be realized for MIMO operation (as shown in [41]). Since the horn-feed with the lens is found to have a high peak realized gain, it can be considered a better choice for MIMO operation, with a down side of higher fabrication cost.

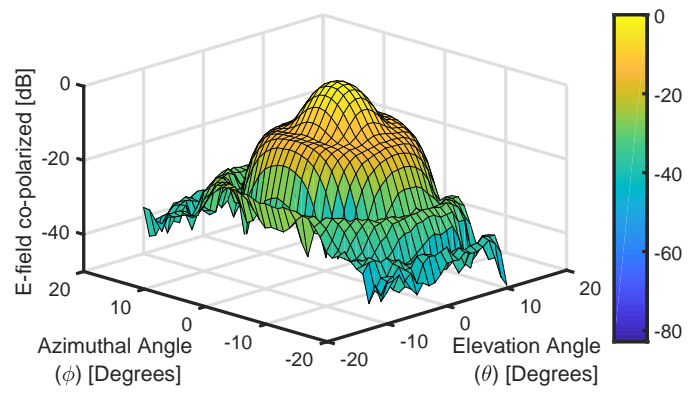

(a)

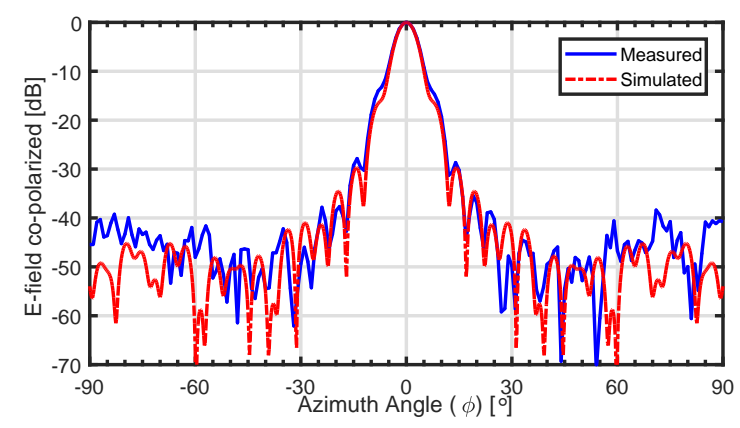

(b)

Fig. 7. (a) Measured 3-D main beam far-field patterns of the constant $-\epsilon_{r}$ lens. (b) Comparison between measured and simulated far-field patterns along the azimuth directions.

\section{Multiuser Performance Evaluations}

\section{A. Channel Simulations}

The attenuation models for the complex path and the DOAs are assumed to be uncorrelated. Here, $\beta_{\ell}=\zeta_{\ell}\left(k_{\text {ref }} / k_{\ell}\right)^{\chi}$ defines the large-scale fading, that involves the shadowing affects and geometric attenuations at the distance $k_{\ell}$ from the $\ell$-th UE to the BS. To further elaborate this, $10 \log _{10}\left(\zeta_{\ell}\right) \sim$ $\mathcal{C N}\left(0, \varsigma_{\mathrm{sf}}^{2}\right)$, where $\varsigma_{\mathrm{sf}}$ is the standard deviation of the fading impact represented by independent and identically distributed (i.i.d.) random entries having complex Gaussian distribution. Also, $\chi$ is the attenuation exponent while $k_{\text {ref }}$ is a reference distance from the BS. We first investigated the impact of the constant $-\epsilon_{r}$ lens beamformer on per-terminal ZF SNR and sum spectral efficiency. For the channel simulations, we assume a sector radius of $K_{\text {sector }}=100 \mathrm{~m}$ with a carrier frequency $f_{c}=28 \mathrm{GHz}$, and a LOS attenuation exponent of 2 . We considered $k_{\text {ref }}=10 \mathrm{~m}$ so that all the UEs are randomly located between $k_{\text {ref }}$ and $K_{\text {sector }}$. The number of UE terminals within the cell sector is considered to be 9 while the noise power at the BS array, $\varsigma^{2}=1$, implying that the $\rho_{t}$ is the average operating SNR. We consider a net angular spread of $70^{\circ}$ in azimuth and elevation for each terminal's DOAs arriving at the BS. Also we considered MPCs $N_{\mathrm{P}}=4$ which can either be distinct or clustered. We simulated 10,000 Monte-Carlo realizations of the small-scale fading, when each realization considered a unique complementary large-scale fading random variable that depends upon the link distance. 


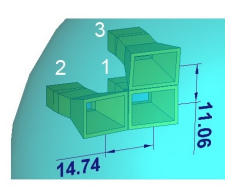

(a)

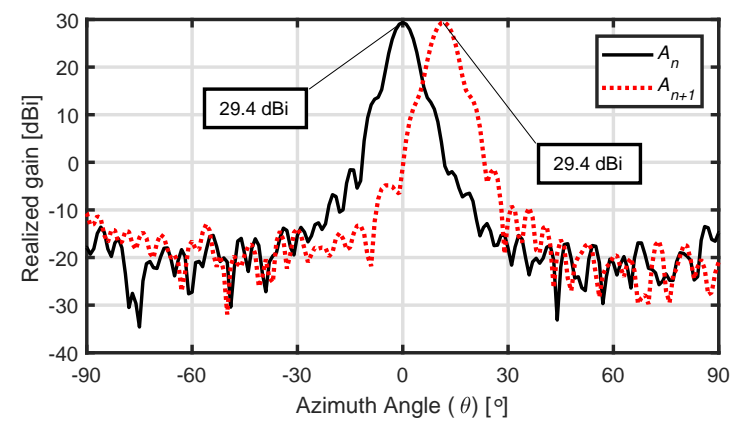

(c)

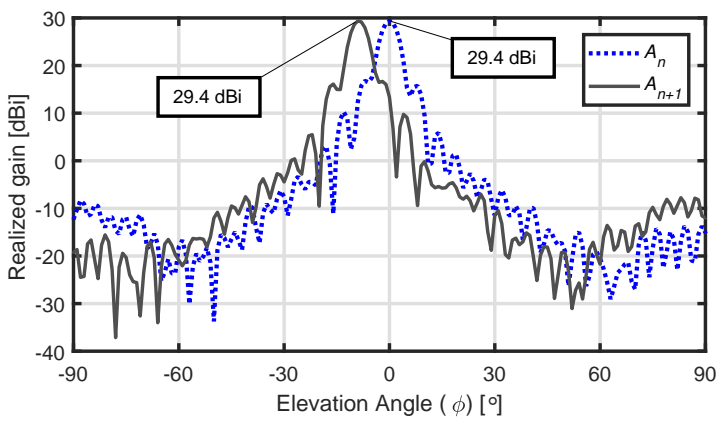

(d)

Fig. 8. (a) Geodesic placement of first three possible horn antenna-feeds on constant $\epsilon_{r}$ lens (all dimensions are in mm) (b) Mutual coupling between three horn antenna-feeds. Realized gain plots comparison in the presence of other two horn antenna-feeds along (c) azimuth and (d) elevation zones.

\section{B. Multi-beam Performance}

Horn antenna-feed with the constant $-\epsilon_{r}$ lens reveals a highly directive beam having a peak gain value of $29.4 \mathrm{dBi}$. Closely spaced antenna elements face mutual coupling, that can be estimated from $S$-parameters (i.e. $\left|S_{21}\right|$ ) and have a negative impact on per antenna element efficiency. The correlation coefficient, on the other hand, is calculated from 2$\mathrm{D}$ radiation patterns [42]. Theoretical model in [43] have also shown that when the propagation is assumed to be uniformly random, correlation coefficient can also be evaluated from $S_{-}$ parameters using:

$$
\rho_{e}=\frac{\left|S_{11}^{*} S_{12}+S_{21}^{*} S_{22}\right|}{\left(1-\left(\left|S_{11}\right|^{2}+\left|S_{21}\right|^{2}\right)\right)\left(1-\left(\left|S_{22}\right|^{2}+\left|S_{12}\right|^{2}\right)\right)} .
$$

The geodesic placement of the elements $\left(A_{n}, A_{n}+1\right.$ and so on) is shown in Fig. 8 (a) where the inter-element pitch along azimuth and elevation is different. For clarity, only three elements are shown with their corresponding mutual coupling can be noted to be $<10 \%$. As discussed before, the resolution of zone coverage is informed by the MU-MIMO sytem requirements, and governs the possibility of the element placement. In this particular case, the beamformer is capable of serving to 8 dominant DOAs in azimuth and 3 in elevation zones, since the realized gain maximas are $12^{\circ}$ and $9^{\circ}$ apart along these zones. In addition to beamforming capabilities, the system reveals high beam de-correlation, corroborated by the fact that the first overlap between two beams occur relatively $12.4 \mathrm{~dB}$ and $9.4 \mathrm{~dB}$ below the peak gain values, along azimuth and elevation zones, respectively. Note that the separation of neighboring beams is governed by the angular spread of the propagation channel, and since it can be different in azimuth and elevation zones [44], having same angular resolution along both the zones is not a compulsion. That is the main reason the angular separation between horn-feed along azimuth and elevation zones is different.

In contrast to the multi-beam operations using similar lens types, the beam separation and corresponding achievable angular resolution in this work makes it a very good choice for sector coverage in mmWave BS scenario. This may not be possible due to physical limitations, for example in [25], where the angular separation can only be $60^{\circ}$. This is in addition to the scalability of the constant $-\epsilon_{r}$ lens because of its capability of focusing energy outside the lens surface, compared to [25], [27] where this is not the case. It is very important to note that the placement of the horn antenna-feeds along both, the azimuth and elevation directions does not change the gain values as can be seen from Fig. $8($ c) and (d). This makes the proposed mmWave MIMO beamformer superior to a number of other topologies, including the phased antenna arrays, where the peak directivity naturally degrades as $\theta$ or $\phi$ increases. Net functionality of a beamformer at the BS is represented in terms of $L \times M$ matrix when:

$$
\mathbf{F}_{\mathrm{RF}}=\left[\mathbf{a}^{H}\left(\phi_{1}, \theta_{1}\right) \mathbf{a}^{H}\left(\phi_{2}, \theta_{2}\right) \ldots \mathbf{a}^{H}\left(\phi_{M}, \theta_{M}\right)\right]^{T} .
$$

In the light of above discussion, here, we assume that the same 2-D measured data (Fig. 7 (a)) translates along the azimuth and elevation zones, corresponding to the horn antenna-feeds at $A_{n}, A_{n+1}$ and so on. A practical system is expected to perform equally well since the accuracy of the measurements and simulation agreement is very much evident from Fig. 7 (b). Horn-feed separation of Fig. 8(a) is one of the choice, optimized for a particular angular separation. Note that the horn-feed aperture can be increased or decreased to compensate the angular resolution along azimuth or elevation zones. This will have a direct impact on maximum allowable beam angular separation and realized gain. Fig. 9 shows the results from a similar comparative study, revealing the tradeoff between horn-feed separation, maximum gain and HPBW. Increased horn-feed pitch would increase the blind spots the array would be seeing the channel in. However, keeping the angular spread of the channel as our basis, if we select the maximum possible gain, equivalent to a particular level of pitch, then the desired signal power would be maximized but the interference would be nulled due to $\mathrm{ZF}$ processing. In the case of closely spaced users, the noise inflation may reduce the overall performance, so a careful consideration of channel is required to efficiently decide pitch and gain of a particular front-end. 


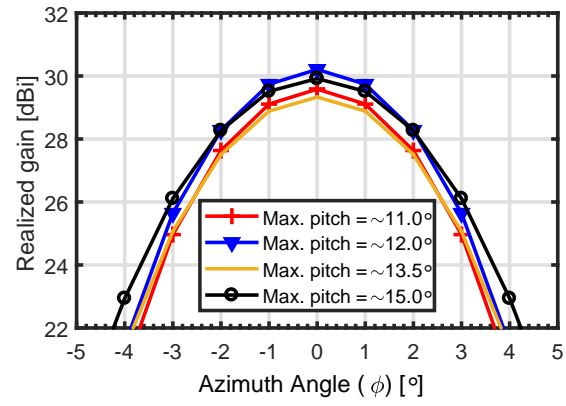

Fig. 9. Impact of maximum allowable angular separation on far-field gain when horn-feed aperture is increased.

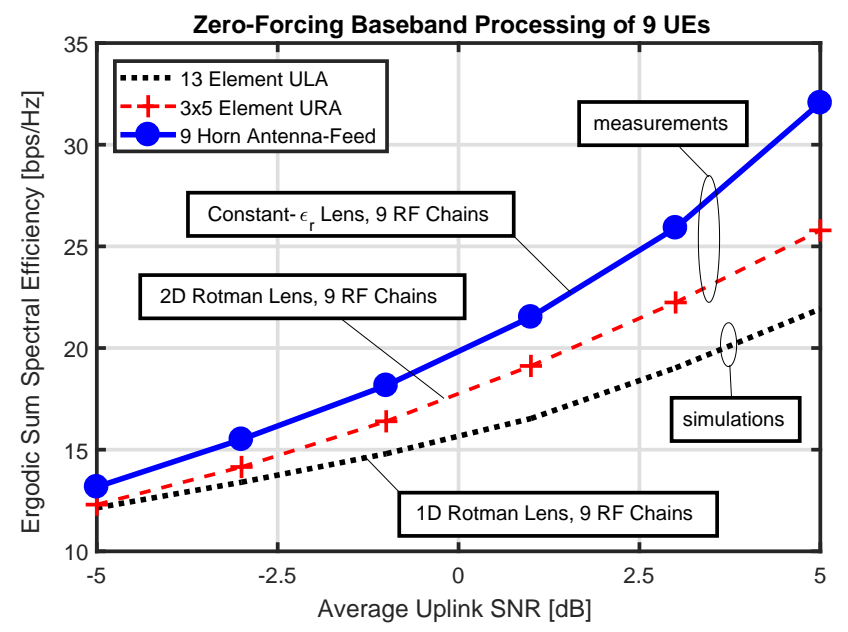

Fig. 10. Ergodic sum rate of the system at an average operating SNR level for with 9 users.

\section{Spectral Efficiency Results}

At the base-band SP, the ZF processing nullifies the multiuser interference, such that the SNR for a given $\ell$-th user becomes:

$$
\mathrm{SNR}_{\ell}=\frac{\rho_{\mathrm{t}}}{\varsigma^{2}\left\{\operatorname{Tr}\left[\left(\mathbf{G}^{H} \mathbf{G}\right)^{-1}\right]_{\ell, \ell}\right\}} .
$$

The ZF SNR for terminal $\ell$ can be projected to give an instantaneous spectral efficiency $\mathrm{R}_{\ell}=\log _{2}\left(1+\mathrm{SNR}_{\ell}\right)$ in $\mathrm{bit} / \mathrm{sec} / \mathrm{Hz}$. This has been used to compute the sum spectral efficiency of the system using:

$$
\mathbf{R}_{\text {sum }}=\sum_{\ell=1}^{L} \mathbf{R}_{\ell} .
$$

In Fig. 10, we evaluated the ergodic sum spectral efficiency of the different topologies. Shadow fading standard deviation is considered to be $6 \mathrm{~dB}$ while the path-loss exponent is 3.4.

As no third party comparative results are available in the literature, $\lambda / 2$ spaced ULA with a non-ideal Rotman lens model is considered as a benchmark to compare with the performance of the constant $-\epsilon_{r}$ lens. Two important trends are observed from the comparison. First, the performance of all three beamformers is almost the same at low SNR values; however, the aggregate impact of constant $-\epsilon_{r}$ lens beamformer compared to Rotman lens based ULA and URA beamformers leads to a significant performance improvement in spectral efficiency at moderate and high $\rho_{t}$ values. Compared to ULA beamformer, the URA beamformer improves the sum rates up to $\sim 2 \mathrm{bps} / \mathrm{Hz}$ at $\mathrm{SNR}=0 \mathrm{~dB}$ due to multi-path diversity added as a result of elevation zone coverage. ULA is limited in the azimuthal spatial degrees of freedom, while more number of active MPCs contributes to recover the terminal's data stream in the case of URA. With an added benefit of covering the elevation zone, the constant $-\epsilon_{r}$ lens beamformer enhance the sum rate $\sim 2 \mathrm{bps} / \mathrm{Hz}$ further. Due to the Rotman lens's spillover losses, URA and ULA beamformers cannot accurately focus the specific DOA to a distinct beam-ports, causing additional interference [34]. While on the other hand, the focusing capability of the constant $-\epsilon_{r}$ lens (see Fig. 3) reduces the interference, assisting the baseband SP to efficiently decorrelate the independent data streams even when the UEs are closely spaced. Secondly, at SNR $=5 \mathrm{~dB}$, constant$\epsilon_{r}$ lens beamformer significantly outperforms both ULA and URA beamformers with an increment of $\sim 6 \mathrm{bps} / \mathrm{Hz}$. This is because the fundamental impairment of the Rotman lens causing EM energy spill-over regardless of the $\rho_{t}$, while high $\rho_{t}$ values assists constant $-\epsilon_{r}$ lens to overcome the dielectric losses within its structure.

Overall, the presented result predicts the system performance of the constant $-\epsilon_{r}$ lens and relates it to the case where perfect Rotman lens based operation is assumed (a routine assumption in the literature). To the best of the authors' knowledge, this is the first time demonstration of constant$\epsilon_{r}$ lens beamformer for mmWave MU-MIMO.

\section{CONCLUSION}

In the paper, we demonstrated the capabilities of a constant$\epsilon_{r}$ lens in an attempt to realize a low complexity RF frontend for mmWave MU-MIMO. With the aid of measurements of the lens beamformer, we predicted the end-to-end system performance of hybrid MU-MIMO architecture in terms of ergodic sum spectral efficiency for 9 UE terminals. To draw a comparison, we used the performance of previously reported classical analog Rotman lens based beamformers connected to ULA and URA. The results depict the superiority of the constant $-\epsilon_{r}$ lens in terms of cost, complexity and performance. The capacity gains acquired with the proposed solution, when coupled to the mechanical and thermal properties of the lens beamformer, suggest that it could provide a useful engineering solution for mm-wave beamforming.

\section{ACKNOWLEDGMENT}

The authors would like to thank W. McKeown and C. Colligan for their assistance in careful machining of the lens and horn-feed. Also, thanks to K. Rainey for helping in the measurements.

\section{REFERENCES}

[1] E. Björnson, E. G. Larsson and T. L. Marzetta, "Massive MIMO: ten myths and one critical question," IEEE Commun. Mag., vol. 54, no. 2, pp. 114-123, Feb. 2016. 
[2] Sadhu. Bodhisatwa, et al., "A 28-GHz 32-element TRX phased-array IC with concurrent dual-polarized operation and orthogonal phase and gain control for 5G communications,' IEEE J. Solid-State Circuits, vol. 52, no. 12 , pp. 3373-3391, Dec. 2017.

[3] J. Ala-Laurinaho et al., "2-D beam-steerable integrated lens antenna system for 5GE-band access and backhaul," IEEE Trans. Microw. Theory Techn., vol. 64, no. 7, pp. 2244-2255, Jul. 2016.

[4] D. W. Prather et al., "Optically upconverted, spatially coherent phasedarray-antenna feed networks for beam-space MIMO in 5G cellular communications," IEEE Trans. Antennas Propag., vol. 65, no. 12, pp. 6432-6443, Dec. 2017.

[5] E. Dahlman et al., "5G wireless access: Requirements and realization," IEEE Commun. Mag., vol. 52, no. 12, pp. 42-47, December 2014.

[6] W. Hong, et al., "Multibeam antenna technologies for 5G wireless communications," IEEE Trans. Antennas Propag., vol. 65, no. 12, pp. 6231-6249, Mar. Jun. 2017.

[7] R. K. Luneburg and M. Herzberger, "Mathematical theory of optics," Univ of California Press, 1964.

[8] H. Chou and Z. Yan, "Parallel-plate Luneburg lens antenna for broadband multi-beam radiation at millimeter wave frequencies with design optimization," IEEE Trans. Antennas Propag., vol. 66, no. 11, pp. 5794 5804, Nov. 2018.

[9] C. Hua, X. Wu, N. Yang and W. Wu, "Air-filled parallel-plate cylindrical modified Luneberg lens antenna for multiple-beam scanning at millimeterwave frequencies," IEEE Trans. Microw. Theory Techn. , vol. 61, no. 1, pp. 436-443, Jan. 2013.

[10] Q. Lin and H. Wong, "A low-profile and wideband lens antenna based on high-refractive-index metasurface," IEEE Trans. Antennas Propag., vol. 66, no. 11, pp. 5764-5772, Nov. 2018.

[11] Ma, Hui Feng and Cui, Tie Jun, "Design of 3-D monolithic MMW antennas using ceramic stereolithography," IEEE Trans. Antennas Propag., vol. 55, no. 3, pp. 1-21, Mar. 2007.

[12] M. Jiang, Z. N. Chen, Y. Zhang, W. Hong and X. Xuan, "Metamaterialbased thin planar lens antenna for spatial beamforming and multibeam massive MIMO," IEEE Trans. Antennas Propag., vol. 65, no. 2, pp. 464472, Feb. 2017.

[13] S. B. Glybovski, et al. "Metasurfaces: From microwaves to visible," Physics Reports, vol. 634, no. 1, pp. 1-72, May. 2016.

[14] H. F. Ma, and T. J. Cui, "Three-dimensional broadband ground-plane cloak made of metamaterials," in Nature communications, vol. 1, pp. 121, Jun. 2010.

[15] S. V. Hum and J. Perruisseau-Carrier, "Reconfigurable reflectarrays and array lenses for dynamic antenna beam control: A review," IEEE Trans. Antennas Propag., vol. 62, no. 1, pp. 183-198, Jan. 2014.

[16] A. Lamminen, et al., "Gain enhanced millimetre-wave beam-switching Rotman lens antenna designs on LCP," in Proc. IEEE EUCAP, May 2017, pp. 2781-2785.

[17] Y. J. Cheng et al., "Substrate integrated waveguide (SIW) rotman lens and its Ka-band multibeam array antenna applications," IEEE Trans. Antennas Propag., vol. 56, no. 8, pp. 2504-2513, Aug. 2008.

[18] Y. Gao, et al., "Rotman lens based hybrid analog-digital beamforming in massive MIMO systems: Array architectures, beam selection algorithms and experiments," IEEE Trans. Veh. Technol., vol. 66, no. 10, pp. 91349148, Oct. 2017.

[19] R. G. Stephen, and R. Zhang, "Millimeter-wave CRAN with lens antenna arrays," in Proc. IEEE GLOBECOM, Dec. 2017, pp. 1-6.

[20] Y. J. Cho, G. Suk, B. Kim, D. K. Kim and C. Chae, "Lens-embedded antenna array for mmWave MIMO: Design and performance," IEEE Commun. Mag. , vol. 56, no. 7, pp. 42-48, Jul 2018.

[21] T. C. Cheston, and E. J. Luoma, "Constant-K lenses," in Emerson \& Cuming, APL Technical Digest, Mars-Avri, Mar 1963.

[22] L. Gunderson, "An electromagnetic analysis of a cylindrical homogeneous lens," IEEE Trans. Antennas Propag., vol. 20, no. 4, pp. 476-479, Jul. 1972.

[23] W. Free, F. Cain, C. Ryan, C. Burns and E. Turner, "High-power constantindex lens antennas," IEEE Trans. Antennas Propag., vol. 22, no. 4, pp. 582-584, July 1974.

[24] Briqech, A. R. Sebak, T. Denidni, "60 GHz circular patch-fed high gain transparent lens antenna", Appl. Comput. Electromagn. Soc. J., vol. 30, no. 5, pp. 469-477, 2015.

[25] Z. Briqech, A. Sebak and T. A. Denidni, "Wide-scan MSC-AFTSA array-fed grooved spherical lens antenna for millimeter-wave MIMO applications," IEEE Trans. Antennas Propag., vol. 64, no. 7, pp. 29712980, July 2016.

[26] Z. Briqech and A. Sebak, "60 GHz microstrip-fed high gain dielectric lens antenna," Proc. CCECE, Apr. 2012, pp. 1-4.

[27] J. M. Jacob, M. Methini and A. Sidhique, "Analysis of dielectric lens loaded antenna," Proc. IEEE ICICCS, Jun. 2017, pp. 959-961.

[28] S. Chen and W. Dou, "Analysis and design of multi-beam antenna with dielectric spherical lens at millimeter-wave band," Proc. China-Japan Joint Microwave Conference, Sept. 2008, pp. 129-132.

[29] C-Lec Plastics Inc., "Rexolite," [Online]. Available: http://www.rexolite.com/ [Accessed Jan. 12, 2018].

[30] Y. Hu et al., "A digital multibeam array with wide scanning angle and enhanced beam gain for millimeter-wave massive MIMO applications," IEEE Trans. Antennas Propag., vol. 66, no. 11, pp. 5827-5837, Nov. 2018.

[31] A. Sayeed and N. Behdad, "Continuous aperture phased MIMO: Basic theory and applications," Proc. Allerton, Sept. 2010, pp. 1196-1203.

[32] R. C. Hansen, "Design trades for Rotman lenses," IEEE Trans. Antennas Propag., vol. 39, no. 4, pp. 464-472, Apr. 1991.

[33] H. Tataria, et al., "Uplink interference analysis with RF switching for lens-based millimeter-wave systems," in Proc. IEEE ICC, May 2018, pp. $1-7$.

[34] M. A. B. Abbasi, H. Tataria, V. F. Fusco and M. Matthaiou, "On the impact of spillover losses in $28 \mathrm{GHz}$ Rotman lens arrays for 5G applications," in Proc. IEEE IMWS-5G, Aug. 2018, pp. 1-3.

[35] M. A. B. Abbasi, H. Tataria, V. F. Fusco and M. Matthaiou, "Performance of a $28 \mathrm{GHz}$ two-stage rotman lens beamformer for millimeter wave cellular systems," in Proc. IEEE EUCAP, Apr. 2019. (submitted).

[36] E. G. Larsson, T. L. Marzetta, H. Q. Ngo, and H. Yang, "Antenna count for massive MIMO: $1.9 \mathrm{GHz}$ versus $60 \mathrm{GHz}$," IEEE Commun. Mag., vol. 56, no. 9, pp. 132-137, Sep. 2018.

[37] J. Ko, et al., "Millimeter-wave channel measurements and analysis for statistical spatial channel model in in-building and urban environments at 28 GHz," IEEE Trans. Wireless Commun., vol. 16, no. 9, pp. 5853-5868, Sep. 2017.

[38] A. F. Molisch, et al., "Hybrid beamforming for massive MIMO: A survey," IEEE Commun. Mag., vol. 55, no. 9, pp. 134-141, Sep. 2017.

[39] M. Katz, Introduction to Geometrical Optics, World Scientific Publishing Company, 2002.

[40] CST Studio Suite by Computer Simulation Technology, Darmstadt, Germany, 2018. [Online] Available: http://www.cst.com.

[41] M. A. B. Abbasi, V. Fusco, H. Tataria, M. Matthaiou, "Lens-based beamformer for low-complexity millimeter-wave cellular systems," Proc. ESA Workshop on Millimetre-Wave Technology and Applications, Dec. 2018.

[42] R. G. Vaughan and J. B. Andersen, "Antenna diversity in mobile communications," IEEE Trans. Veh. Technol., vol. 36, no. 4, pp. 149172, Nov. 1987.

[43] S. Blanch, J. Romeu and I. Corbella, "Exact representation of antenna system diversity performance from input parameter description," Electron. Lett., vol. 39, no. 9, pp. 705-707, 1 May 2003.

[44] "Study on channel model for frequencies from 0.5 to $100 \mathrm{GHz}$," 3GPP, Sophia Antipolis, France, Rep. TR 38.901 V14.3.0, Dec. 2017. 